\title{
Laparoscopic Heminephrectomy for Benign Renal Anomalies
}

\author{
Justin Zumsteg, B.S., William W. Roberts, M.D., and J. Stuart Wolf, Jr., M.D.
}

\begin{abstract}
Background and Purpose: Benign renal conditions, such as duplication and fusion anomalies, are relatively common and may lead to problems such as chronic flank pain or urinary tract infection. Traditional management of these conditions has been open surgical removal of the affected moiety. As an alternative, we report on our experience with laparoscopic heminephrectomy for benign renal anomalies and emphasize the technical aspects of the procedures.

Patients and Methods: We retrospectively reviewed the records of patients who underwent laparoscopic heminephrectomy for benign renal anomalies at our institution between February 1999 and April 2009.

Results: Heminephrectomy was performed in 11 patients, including 8 with duplicated collecting systems, 2 with horseshoe kidneys, and 1 with a cross-fused renal ectopic kidney. Median operative time was 204 minutes, and median estimated blood loss was $250 \mathrm{~mL}$. There were no intraoperative complications. The presenting symptoms resolved in all patients. There were three major and two minor postoperative complications, including 50\% loss of remaining ipsilateral renal parenchyma, postoperative neuralgia and anejaculation, urinoma, and partial wound separation. Median length of hospital stay was 2 days. Renal function was well preserved in all patients. The median change in serum creatinine level was $0.1 \mathrm{mg} / \mathrm{dL}$.

Conclusions: Although laparoscopic heminephrectomy is challenging because of the variable blood supply and abnormal anatomy of the kidney, with careful planning and attention to detail, the procedure is safe and effective for the management of a wide range of benign renal anomalies that necessitate heminephrectomy and offers the usual recovery advantages of laparoscopy.
\end{abstract}

\section{Introduction}

E RRORS IN GENITOURINARY TRACT DEVELOPMENT make benign renal conditions, such as duplication and fusion anomalies, relatively common. Ureteral duplication is the most frequent duplication anomaly, present in 1 in 125 births. ${ }^{1}$ Duplicated ureters are frequently associated with an obstructed upper-pole moiety or a refluxing lower-pole moiety. Either abnormality can produce atrophy of the moiety and the development of clinical problems, such as flank pain and recurrent urinary tract infections (UTIs). Horseshoe kidney and cross-fused renal ectopia represent the most common fusion anomalies, occurring at rates of 1 in 400 and 1 in 2000 live births, respectively. ${ }^{2,3}$ While many of these patients are asymptomatic, these anomalies are associated with higher rates of ureteropelvic junction (UPJ) obstruction and nephrolithiasis than in normal kidneys and, as such, are more likely than in normal kidneys to cause signs or symptoms that necessitate surgical intervention.

Laparoscopic techniques for the surgical management of each of these entities have been described previously, but the majority of these reports are in the pediatric literature or, in adults, are only case reports. The first description of a laparoscopic heminephrectomy for benign renal anomaly was in 1993 by Jordan and Winslow, ${ }^{4}$ in a child. It was not until 2007 that a case series of exclusively adult patients who were undergoing laparoscopic heminephrectomy was published, when Aboussaly and coworkers ${ }^{5}$ reported a series of five laparoscopic upper-pole heminephrectomies for duplicated ureters.

In this article, we review our experience with laparoscopic heminephrectomy for benign renal anomalies in adults, including, to our knowledge, the largest series of laparoscopic heminephrectomy for symptomatic ureteral duplications in adults. We provide a detailed review of the surgical technique used and report on our operative experience, complications, and postoperative results.

\section{Patients and Methods}

After obtaining Institutional Review Board approval, a retrospective review was performed of our database that

Department of Urology, University of Michigan Health System, Ann Arbor, Michigan. 
identified 11 patients who had undergone laparoscopic heminephrectomy for benign renal anomalies between February 1999 and April 2009.

Patients are prepared for surgery with magnesium citrate bowel preparation and preoperative administration of antibiotics. In some cases, we cystoscopically place a ureteral catheter in the unaffected ureter at the outset of the procedure under fluoroscopic control, with retrograde pyelography confirming placement of the end of the $6 \mathrm{~F}$ ureteral catheter in the renal pelvis. This catheter is used to aid in identification of the unaffected ureter and to inject dyed saline to assess for collecting system entry. The ureteral catheter is removed at the conclusion of the procedure.

For transperitoneal laparoscopy, the patient is placed in a partial lateral decubitus position ( 45 degrees from horizontal) with careful padding. The table is flat, without flexion. For standard transperitoneal laparoscopy, transabdominal access is attained with a Veress needle, and insufflation is with carbon dioxide at $15 \mathrm{~mm} \mathrm{Hg}$ pressure. Dilating (noncutting) laparoscopic ports are inserted, including a 12-mm port for the video-laparoscope, one working 12-mm port (if larger instrumentation, such as clip applier, stapler, or laparoscopic ultrasonography probe are needed), and two to three additional 5-mm ports for retraction and other instruments. In the most recent cases, a 5-mm laparoscope via a $5-\mathrm{mm}$ port was used. Port placement is generally the same as that for nephrectomy.

For hand-assisted transperitoneal laparoscopy, the handassistance device is placed through an 8- to 9-cm incision. Ports include two $12-\mathrm{mm}$ ports and one $5-\mathrm{mm}$ port. We used this approach only for horseshoe kidneys. Because a horseshoe kidney is lower and more medial in the abdomen than a normal kidney, the hand assistance incision is made in the lower midline or through a Pfannenstiel incision, and ports need to be moved accordingly.

The kidney is exposed by incising along the line of Toldt and reflecting the colon medially. The liver or spleen is retracted as necessary. The affected portion of the kidney is identified using visual clues, palpation with the laparoscopic instruments, and laparoscopic ultrasonography, as needed. In cases with a dilated ureter (heminephrectomy for obstructed or refluxing moiety), it is easier to identify the dilated ureter first and trace this up to the kidney. In cases of an obstructed upper-pole moiety, the affected ureter passes under the main renal vessels and must be meticulously freed from these structures.

Division of the ureter includes occlusion if the ureter refluxes or drains externally, and the ureter is left open if the ureterovesical junction is obstructed. The renal arteries and veins associated with the affected segment are controlled with bipolar cautery if small and with clips or staples if larger. The margin of the affected portion of the kidney with the normal kidney is completely dissected.

Once the affected segment is exposed by complete dissection and its vasculature occluded, the ureter is used to help retract the segment away from the remaining (healthy) kidney. The renal tissue is excised using laparoscopic scissors, with or without cautery, depending on the clarity of the demarcation between the affected and normal portions of the kidney, or with bipolar scissors, ultrasonic shears, or stapler. Any bleeding from the resection surface is controlled with argon beam coagulation, gelatin-thrombin matrix, and/or sutures. If there is any question as to the integrity of the collecting system, dyed saline is injected through the preplaced ureteral catheter. The laparoscopic ports are removed, and the fascia and wounds closed. A drain is left only if there is concern about collecting system leakage.

For a retroperitoneoscopic approach, the patient is placed in a full flank position, and the table is flexed with the kidney rest elevated. A $20-\mathrm{mm}$ incision is made one finger breadth below the 12th rib. The retroperitoneum is entered by blunt dissection down to the lumbodorsal fascia, which is opened with insertion of a clamp. Digital dissection expands the space posterior to the kidney along the psoas muscle. A balloon dilator directed along the psoas muscle posterior to the kidney expands the retroperitoneal space. A 12- $\mathrm{mm}$ balloon port is used for the camera port at this site, and the retroperitoneal space is insufflated to $15 \mathrm{~mm} \mathrm{Hg}$ with carbon dioxide. A 5-mm port is positioned two finger breaths above the iliac crest, and medial to the camera port. A $12-\mathrm{mm}$ port is placed at the junction of the 12th rib and the spinous musculature.

Intraoperative complications were defined as complications occurring during the course of surgery that caused a change in postoperative management, including blood transfusions. Postoperative complications were assessed during hospitalization and in the 3 months postoperatively, although even later developments were considered postoperative complications if they were related to the surgery. Major and minor complications did or did not, respectively, necessitate major intervention or readmission. Data were obtained from our laparoscopy database, supplemented by review of the medical record and phone contact of the patients and/or referring physicians. No statistical analyses were performed.

\section{Results}

Patient data are summarized in Table 1. Patient ages ranged from 20 to 42 years (mean $30.1 \mathrm{yr}$ ). Of the patients, five had an American Society of Anesthesiologists score of 1 and six had a score of 2. Surgery was performed on the right side in six patients and the left side in five patients. Of the 11 patients, 8 underwent removal of a duplicated collecting system (6 upper pole and 2 lower pole), 2 underwent removal of a portion of a horseshoe kidney, and 1 underwent removal of the crossed portion of a cross-fused ectopic kidney. The excised segment had minimal function, assessed by cross-sectional imaging that revealed very thin parenchyma in all cases and was confirmed by nuclear medicine renal scan in one case.

Of the patients, nine presented with symptoms. In four of the six patients with abnormal upper-pole moieties, the underlying problem was distal ureteral obstruction (Fig. 1). All four of these patients presented with flank pain, and two had recurrent UTIs. The remaining two patients with abnormal upper-pole moieties both had ectopic insertion of the affected ureter into the vagina; both presented with urinary incontinence, and one also experienced recurrent UTIs. Of the two patients with excision of lower-pole moieties of duplicated collecting systems, one had recurrent UTIs (associated with vesicoureteral reflux) and the other reported flank pain and recurrent UTIs (from a large renal stone associated with UPJ obstruction, Fig. 2). 


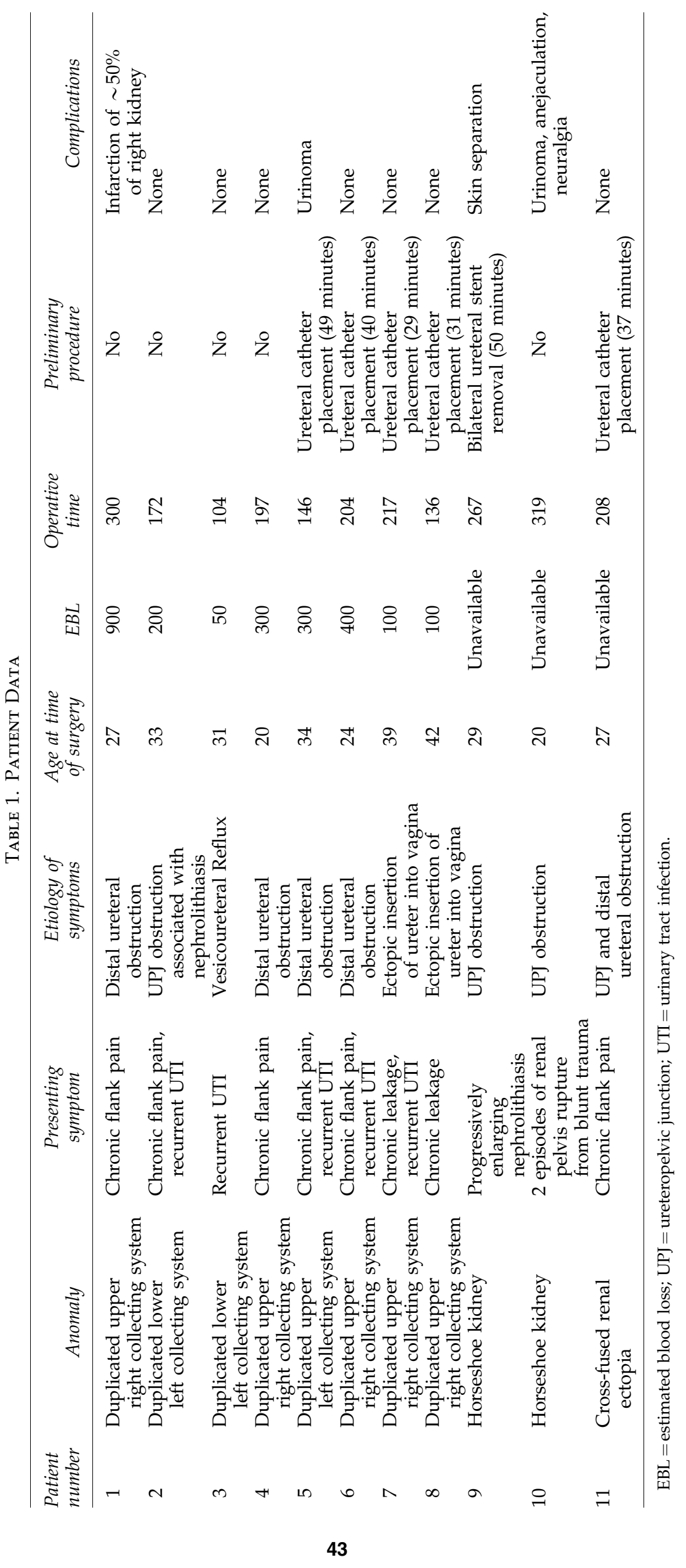




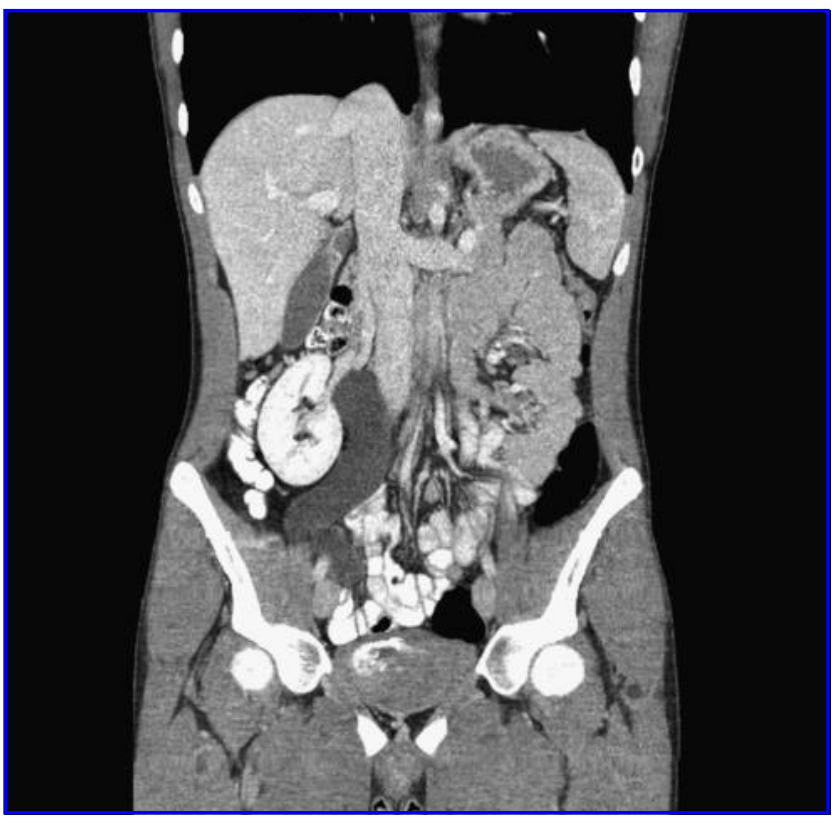

FIG. 1. Patient 4. Coronal formatted CT with intravenous contrast reveals unopacified and dilated ureter to upper-pole moiety.

Finally, the patient who underwent removal of the crossed portion of a cross-fused ectopic kidney appeared to have both UPJ and distal ureteral obstruction (Fig. 3), and reported chronic flank pain. Overall, then, presenting symptoms included chronic flank pain alone in three patients, recurrent UTIs plus flank pain in three patients, recurrent UTIs alone in one patient, and urinary incontinence in two patients (one also with recurrent UTIs).

In both patients with a horseshoe kidney, one moiety was affected by UPJ obstruction. Neither was symptomatic at the time of surgery, but one patient had two episodes of rupture of the renal pelvis from blunt trauma and the other had progressively enlarging renal stones.

Median operative time was 204 minutes (mean 206 minutes, range $104-319 \mathrm{~min}$ ). This does not include retrograde ureteropyelography and ureteral catheter placement in five patients and bilateral stent removal in one patient (procedure time 29-50 minutes, mean 39 minutes). Ureteral catheters were placed in four of the six patients with abnormal upperpole moieties and in the patient with crossed-fused ectopia. The median estimated blood loss was $250 \mathrm{~mL}$ (mean 294 minutes, range 50-900 mL). The median length of hospital stay was 2 days (mean $2.1 \mathrm{~d}$, range $1-5 \mathrm{~d}$ ).

A transperitoneal approach was used in 10 of the patients, while a retroperitoneal approach was used in the case of the cross-fused renal ectopia. The eight operations for duplicated systems were all performed with standard transperitoneal laparoscopy. The resected specimen was removed through the $12-\mathrm{mm}$ port site in six of the cases and was removed from an incision extended to $3 \mathrm{~cm}$ in either the paramedian or Pfannensteil locations in the other two cases. For the eight cases on duplicated collecting systems, two 12-mm ports were used for two of the cases while the rest used only one 12-mm port. In two cases, we used two 5-mm ports, in four we used

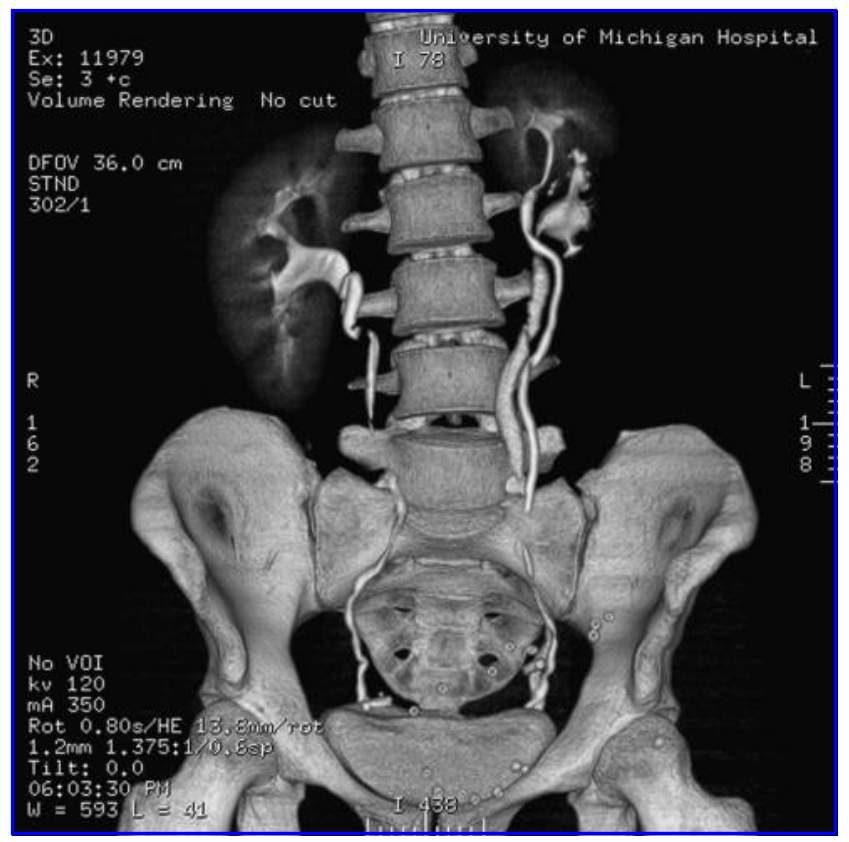

FIG. 2. Patient 2. Three-dimensional formatted CT with intravenous contrast demonstrates abnormal left lower-pole renal moiety with ureteropelvic junction obstruction and minimal renal parenchyma.

three 5-mm ports, and in two we used four 5-mm ports. The total number of ports, then, was three in one case, four in four cases, and five in three cases.

Both resections involving the horseshoe kidneys were performed with hand-assisted laparoscopy, and the speci-

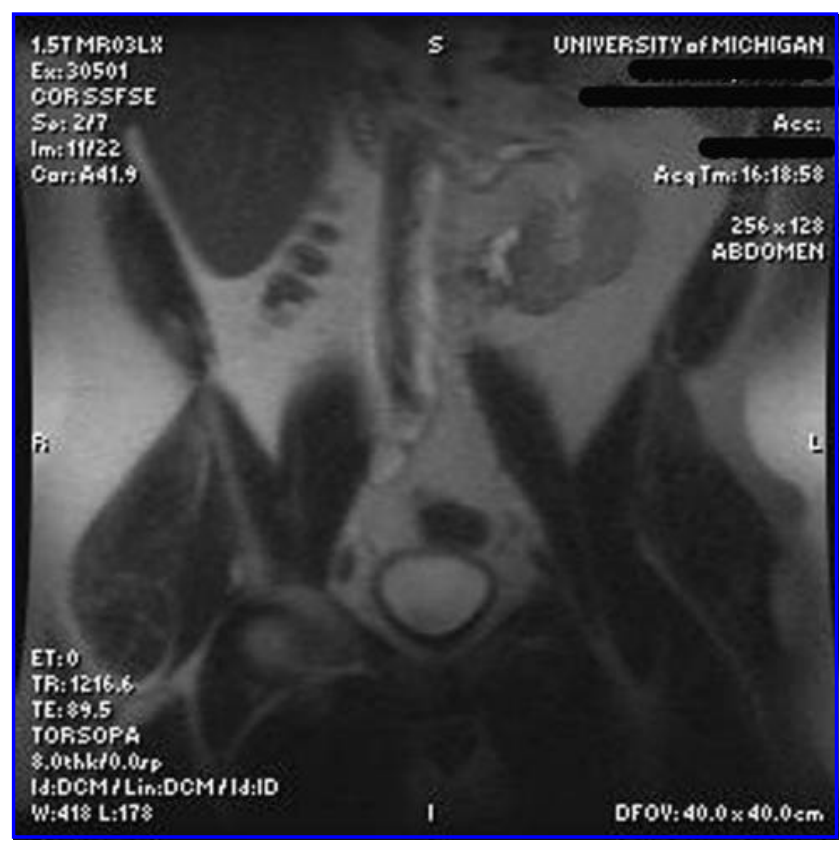

FIG. 3. Patient 11. MRI (coronal format) shows cross-fused renal ectopia with dilation of the ureter to the lower (crossed) moiety and minimal renal parenchyma. 
mens were removed through the hand-assistance incisions of 8 and $9 \mathrm{~cm}$. For the single retroperitoneoscopic case, removal of the specimen was through enlargement of the primary port site (off the tip of the 12th rib) to $3.5 \mathrm{~cm}$. This procedure and the two hand-assisted procedures for horseshoe kidneys used two 12-mm ports and one 5-mm port.

There were no intraoperative complications. The collecting system of the remaining kidney was intact in all cases assessed. There were three major and two minor postoperative complications. Patient 1 (upper-pole heminephrectomy) experienced infarction of approximately $50 \%$ of the remaining ipsilateral renal parenchyma as assessed by CT. This complicated the patient's postoperative course and necessitated a 4-day hospitalization because of fever and nausea that resolved without additional intervention. Kidney function appeared excellent in this patient with a serum creatinine value of $0.8 \mathrm{mg} / \mathrm{dL}$ on postoperative day 15 . This patient declined further imaging. Patient 10 (heminephrectomy of horseshoe kidney) experienced postoperative neuralgia and anejaculation. The anejaculation spontaneously resolved. The neuralgia was controlled through pharmacologic treatment and nerve blocks, and gradually resolved as well.

Urinomas developed in patients 5 and 10. The urinoma in patient 5 (upper-pole heminephrectomy) developed because of obstruction of the normal ipsilateral ureter. During the procedure, a ureteral catheter had been placed and then removed at the conclusion of the procedure. She was discharged on postoperative day 1 in good condition, but on postoperative day 4 , she presented with pain. Imaging revealed a urinoma. Retrograde uretero-pyelography suggested ureteral edema. Placement of a ureteral stent led to resolution of the symptoms. This stent was removed 7 days later, and the patient has since remained pain free. In patient 10 , the source of the urine leak was the stump of the remaining isthmus of the horseshoe kidney (a ureteral catheter had not been placed in this patient). Symptoms of abdominal pain and fever resolved over the course of 4 days with no other intervention necessary. Patient 11 (heminephrectomy of cross-fused renal ectopia) experienced skin separation involving the partial length of one incision site, which did not necessitate antibiotics or other treatment.

Kidney function was well preserved in all patients. Preoperative serum creatinine values were compared with postoperative values for 10 of the 11 patients. A 2-week postoperative value was used for seven of the patients, and a value between 3 and 27 months postoperative was used for the remaining three patients. The median change in serum creatinine level was $0.1 \mathrm{mg} / \mathrm{dL}$ (range 0.1 to $+0.3 \mathrm{mg} / \mathrm{dL}$ ). The preoperative serum creatinine value was not available for patient 1 , so this patient was excluded from analysis. This patient did experience, as noted above, an infarction of approximately $50 \%$ of the remaining renal parenchyma on the symptomatic side. The 2 -week postoperative serum creatinine level was $0.8 \mathrm{mg} / \mathrm{dL}$, however, indicating the presence of excellent kidney function.

All nine symptomatic patients had resolution of their presenting symptoms. Of note, patient 1 complained of chronic right lower-quadrant pain in addition to chronic right flank pain. The patient was counseled preoperatively that upperpole heminephrectomy was not likely to address the pain in the right lower quadrant. While this patient did experience resolution of the flank pain, the right lower-quadrant pain persisted. At the patient's urging, a distal ureterectomy was performed, which did not lead to significant improvement. At this point, the patient was referred to a chronic pain specialist for treatment of residual pain.

\section{Discussion}

Abnormal development of the genitourinary tract may result in duplicated collecting system, fusions, and other anomalies. The abnormal segment of the kidney is prone to dysfunction because of obstruction, reflux, or abnormal drainage and may become symptomatic, most often manifesting as recurrent UTIs or chronic flank pain. Resection of the abnormal moiety (if atrophic, as is usually the case) remains the treatment of choice. This has traditionally been performed through an open surgical approach. As laparoscopy has evolved, however, it has been applied to these conditions. While there have been a number of case series presented in the pediatric literature regarding laparoscopic heminephrectomy, ${ }^{6-13}$ the same is not true for the adult population. To date, there has been only one case series of laparoscopic heminephrectomy for duplicated collecting system in an exclusively adult population, ${ }^{5}$ two for laparoscopic heminephrectomy in a horseshoe kidney, ${ }^{14,15}$ and none for crossfused renal ectopia. The remaining literature consists of individual case reports.

The first step in a successful procedure is a careful preoperative work-up to confirm the diagnosis and define the anatomy. Either CT or MRI (with and without contrast material) can demonstrate the duplicated collecting system and the renal vasculature. Depending on the surgeon's needs, specific angiography can be obtained to better delineate the abnormal renal arteries and veins. Confirmation of poor function in the affected moiety may be obtained with a nuclear medicine renal scan, but it is often unnecessary with clear radiographic evidence of markedly atrophic renal parenchyma. Voiding cystography may be useful to exclude reflux in the ureter that is to be transected.

This can be a challenging procedure because of the variable vasculature and abnormal anatomy, but careful attention during a few key steps facilitates a successful operation. First, it is critical to accurately differentiate the ureter to be transected from the functional ureter, and to use careful dissection to avoid devascularizing the latter. For this reason, we recommend placement of a ureteral catheter as the first step of the procedure in all resections of the upper pole of a duplicated collecting system, because the two ureters cross one another and therefore the normal ureter may be at increased risk. We did this in our last four resections of an upper-pole duplicated moiety, as well as in the case of cross-fused ectopia. Fortunately, despite not using a ureteral catheter in our first two cases of resection of an upper-pole moiety we avoided ureteral damage or urinary leakage.

Next, a meticulous dissection of the hilum will aid in correctly identifying the vasculature to the atrophic moiety and in avoiding devascularization of the functional moiety. Finally, defining the plane between the atrophic moiety and the healthy renal parenchyma will minimize the risk of bleeding as well as decrease the incidence of urinoma because of residual parenchyma. The plane is defined both by 
distinguishing the color of the healthy parenchyma from that of the devascularized and atrophic tissue, and by palpating with laparoscopic instruments or the intra-abdominal hand (in hand-assisted cases).

All of our major postoperative complications are illustrative of important points regarding these procedures. In patient 1 , with loss of $50 \%$ of functional parenchyma, there were four sets of renal arteries and veins noted. While only the uppermost vessels were clipped, it is thought that trauma to the remaining vessels during dissection led to the infarction. This emphasizes the importance of a delicate dissection of the renal hilum (this was the first case in our series). Although there was loss of some renal parenchyma, this patient has retained excellent kidney function. Patient 10 had neuralgia and anejaculation. The isthmus of this horseshoe kidney was particularly thick and was immediately covering the pelvic neural plexus. It is thought that this plexus was traumatized during dissection, thus leading to the transient symptoms of neuralgia and anejaculation. The anejaculation resolved over a number of months, and the neuralgia was controlled through oral medications and percutaneous nerve blocks until resolution 2 years later. The inferior and medial position of the isthmus of the horseshoe kidney necessitates exposure of anatomy rarely seen during laparoscopic renal surgery (this was the third case in our series). The development of a urinoma in patient 5 would have been prevented if we had left an internal ureteral stent in place in response to the "tight" ureter. Finally, we should point out that the remaining ureteral stumps remained asymptomatic and were without complication in all patients, even those with distal ureteral obstruction.

The results of our eight patients with duplicated collecting systems are similar to those reported by Aboussaly and associates $^{5}$ in 2007 . The mean operative time in our series was 187 minutes (range 104-300 $\mathrm{min}$ ) compared with 189 minutes (range 150-225 $\mathrm{min}$ ) in that report. It is reassuring that similar results are being obtained at different institutions.

Renal anomalies necessitating heminephrectomy more commonly present in the pediatric rather than in the adult population. For this reason, there is a greater volume of literature regarding the experience with laparascopic heminephrectomy in children. It is difficult to draw many conclusions on the relative differences of the procedure in these populations, given the relatively small sizes of the series. A 2005 report by Wallis and associates, ${ }^{13}$ however, noted that 4 of 22 patients who were undergoing retroperitoneal laparoscopic heminephrectomy needed conversion to open surgery and that 2 of 22 had complete loss of function in the remaining moiety. In contrast, the procedures for none of the patients in our series had to be converted to open surgery, and only one patient had a significant injury to the remaining moiety $(50 \%$ loss in one patient). Given the more favorable outcomes in our series and that of Aboussaly and coworkers, ${ }^{5}$ perhaps the delicate renal vasculature in the pediatric patient needs to be considered in the decision between laparoscopic and open surgery for this indication.

\section{Conclusion}

Laparoscopic heminephrectomy is safe and effective for the management of a range of benign renal anomalies necessitating heminephrectomy. Laparoscopy offers several advan- tages over the traditional open approach, including improved visualization intraoperatively, reduced duration and intensity of convalescence, and a superior cosmetic result. Although the laparoscopic technique is challenging because of the variable blood supply and abnormal anatomy, with a clear surgical plan and attention to detail, the laparoscopic procedure is associated with high success and acceptable morbidity.

\section{Note Added in Proof}

Since the submission of this manuscript, we have performed one additional procedure. A 39-year-old woman presented with right flank discomfort and a palpable mass. Imaging revealed a large cystic structure involving the lower pole of the right kidney. We performed transperitoneal standard laparoscopic excision of a lower pole renal moiety with UPJ obstruction using three 5-mm ports and one 12-mm port. Cystoscopic insertion of a ureteral catheter and repositioning took 43 minutes, and the uncomplicated laparoscopic procedure took 151 minutes. The specimen was removed through the 12-mm umbilical port without enlargement. The EBL was $200 \mathrm{ml}$, the hospital stay was 2 days, and at 2 week follow-up there have been no post-operative complications.

\section{Disclosure Statement}

Justin Zumsteg, B.S.: No competing financial interests exist. William W. Roberts, M.D.: Consultant for Terumo Corporation.

J. Stuart Wolf, Jr, M.D.: Consultant for Terumo Corporation; lecturer for Gyrus-ACMI.

\section{References}

1. Schlussel RN, Retik AB. Ectopic ureter, ureterocele and other anomalies of the ureter. In: Wein AJ, Kavoussi LR, Novick AC, Partin AW, Peters CA. Campbell-Walsh Urology. 9th ed. Philadelphia: Saunders Elsevier, 2007, pp 3413.

2. Bauer SB. Anomalies of the upper urinary tract. In: Wein AJ, Kavoussi LR, Novick AC, Partin AW, Peters CA. CampbellWalsh Urology. 9th ed. Philadelphia: Saunders Elsevier, 2007, pp 3283.

3. McDonald JH, McClellan DS. Crossed renal ectopia. Am J Surg 1957;93:995-1002.

4. Jordan GH, Winslow BH. Laparoendoscopic upper pole partial nephrectomy with ureterectomy. J Urol 1993;150:940-943.

5. Aboussaly R, Gill IS, Kaouk JH. Laparoscopic upper pole partial nephrectomy for duplicated renal collecting systems in adult patients. Urology 2007;69:1202-1205.

6. Janetschek G, Seibold J, Radnayr C, Bartsch G. Laparoscopic heminephroureterectomy in pediatric patients. I Urol 1997; 158:1928-1930.

7. Robinson BC, Snow BW, Cartwright PC, et al. Comparison of laparoscopic versus open partial nephrectomy in a pediatric series. J Urol 2003;169:638-640.

8. El-Ghoneimi A, Farhat W, Bolduc S, et al. Retroperitoneal laparoscopic vs open partial nephroureterectomy in children. BJU Int 2003;91:532-535.

9. Gundeti MS, Ransley PG, Duffy PG, et al. Renal outcome following heminephrectomy for duplex kidney. J Urol 2005; 173:1743-1744.

10. Lee RS, Retik AB, Borer JG, et al. Pediatric retroperitoneal laparoscopic partial nephrectomy: Comparison with an age matched cohort of open surgery. I Urol 2005;174:708712 . 
11. Piaggio L, Franc-Guimond J, Figueroa TE, et al. Comparison of laparoscopic and open partial nephrectomy for duplication anomalies in children. J Urol 2006;175:2269-2273.

12. Castellan M, Gosalbez A, Carmack AJ, et al. Transperitoneal and retroperitoneal laparoscopic heminephrectomy-what approach for which patient? J Urol 2006;176:2636-2639.

13. Wallis MC, Khoury AE, Lorenzo AJ, et al. Outcome analysis of retroperitoneal laparoscopic heminephrectomy in children. J Urol 2006;175:2277-2282.

14. Nouri-Mahdavi K, Izadpanahi MH. Laparoscopic heminephrectomy in horseshoe kidney using bipolar energy: Report of three cases. I Endourol 2008;22:667-670.

15. Yohannes P, Dinlenc C, Liatsikos E, et al. Laparoscopic heminephrectomy for benign disease of the horseshoe kidney. ISLS 2002;6:381-384.
Address correspondence to: J. Stuart Wolf, Jr., M.D.

Department of Urology University of Michigan Health System 1500 East Medical Center Drive, TC 3875

Ann Arbor, MI 48109-5330

E-mail: wolfs@umich.edu

\section{Abbreviations Used}

$\mathrm{CT}=$ computed tomography

$\mathrm{MRI}=$ magnetic resonance imaging

$\mathrm{UPJ}=$ ureteropelvic junction

$\mathrm{UTI}=$ urinary tract infection 
